\title{
Cardiac Tachyarrhythmia Detection by Poincaré Plot-Based Image Analysis
}

\author{
Guadalupe Garcia-Isla ${ }^{1}$, Valentina Corino ${ }^{1}$, Luca Mainardi $^{1}$ \\ ${ }^{1}$ Department of Electronics, Information and Bioengineering (DEIB), Politecnico di Milano, Milan, \\ Italy
}

\begin{abstract}
Tachyarrhythmia detection through RR interval analysis could improve performance of monitoring devices. In this paper a Poincaré plot-based image approach is presented. Three cardiac rhythms were analyzed in this study: normal sinus rhythm (NSR), atrial fibrillation $(A F)$ and atrial bigeminy $(A B)$. Using different MIT-BIH databases, 27955, 3363 and 76 images were generated for NSR, $A F$ and $A B$ respectively using a 2-minute window with $50 \%$ overlap. The $80 \%$ of the data available for each rhythm was used to create a reference rhythm image atlas. The remaining $20 \%$ was classified into one of the three categories using mutual information. The process was iterated 10 times, in which images used to construct the atlas and used to create the test set were randomly selected. $A F$ was correctly classified $94.12 \% \pm 0.45, A B 72.00 \% \pm 11.24$ and NSR $80.70 \% \pm 0.54$. The results of the present study suggest that Poincaré plot-based image analysis is a promising path for classifying different rhythms using only ventricular activity.
\end{abstract}

\section{Introduction}

Cardiac tachyarrhythmias encompass a wide heterogeneous group of electrophysiological disorders of both ventricular and supraventricular nature. Although atrial tachyarrhythmias do not represent a direct life-threatening condition, they are coupled with an increased risk of thromboembolic events and with increased morbidity and mortality levels [1]. Atrial fibrillation (AF) exhibits the highest prevalence among all tachyarrhythmias, representing the $90 \%$ of them and affecting 1-2\% of global population [2]. The correct characterization of their burden is of major importance for the application of the necessary treatments and the prevention of these late events.

However, atrial activity analysis can be challenging due to the low amplitude of atrial signals, more susceptible to noise, and the presence of ventricular activity, of greatest amplitude. Computationally expensive methods for ventricular cancellation as well as good quality signals are required for their analysis. In contrast, analysis of cardiac rhythm relying on $R$ peak detection is more robust against noise and easier to perform due to the higher amplitude of QRS complexes in electrocardiogram (ECG) signals.

Long-term monitoring devices as implantable loop recorders with low computational capacity due to battery and memory restrictions, or single-lead external monitoring devices without capacity for acquiring good quality signals, typically rely on rhythm parameters for the detection of arrhythmias [3]. These devices have been ratified to be a powerful tool for detecting and monitoring AF episodes. However, they often fail to capture short paroxysmal AF episodes and other relevant rhythms as atrial flutter or paroxysmal supraventricular tachycardia. This inability to properly differentiate among different types of rhythms is also a cause of frequent false positives [?], triggered as well by frequent ectopic beats. Increased false positives rates constitute a major problem regarding constant monitoring devices, as they generate huge amount of data in need of manual revision.

Poincaré plot analysis, describing each $R R$ interval versus the previous one, has been widely used to classify different rhythms. Previous studies were based on the definition of parameters and thresholds describing particular patterns in the plot [4-9]. However, some patterns either cannot be easily described by simple parameters or cannot be intuitively appreciated. In order to avoid parameter and threshold selection, a method based on the creation of images from Poincaré plot is presented in this paper, investigating the possibility of classifying 2-min ECG recordings using images derived from the plot.

\section{Methods}

\subsection{Data}

Three different public databases available at PhysioNet were used in this project. The MIT-BIH arrhythmia database (MITDB), containing 48 ambulatory half-hour ECG recordings, the MIT-BIH atrial fibrillation database (AFDB) which contains 26 10-hour ambulatory recordings and the long-term atrial fibrillation database (LTAFDB) which contains 84 two channel recordings of around 24 
to 25 hours each [10]. All databases contained rhythm and beat annotations. From the AFDB only AF segments were included in the study. From the MITDB and LTAFDB instead, AF, NSR and AB segments were used. All other rhythms were not analyzed because either too short episodes or too few patients were present. Only one lead was used from all ECG recordings of all databases. As only $R R$ intervals were of our interest, the differences between sampling frequencies and amplitude ranges among databases were not significant.

\subsection{Signal preprocessing}

Signals were filtered by FIR low pass filter of order 64 and cut off frequency of $50 \mathrm{~Hz}$ and a Butterworth high pass filter of order 4 and cut off frequency $0.5 \mathrm{~Hz}$ to eliminate electromyographic noise, baseline wander and power-line interference. Following, R peaks were detected on each signals using Pan Tompkins' algorithm implemented in Matlab [11]. $R R$ intervals were then computed for all signals.

\subsection{Image generation}

For each ECG signal a sliding window of 2 minutes with $50 \%$ overlap was used for the generation of Poincaré plots from the previously computed $R R$ intervals. Each Poincaré plot was treated as a $2 \mathrm{D}$ histogram where the bin size was set to $20 \mathrm{~ms}$ and was then converted into an image using as pixel dimensions the mentioned bin size. For all images a range was fixed between 0 and $1600 \mathrm{~ms}$, yielding a set of images with the same number of pixels independently of the ECG segment rhythm and of the original signal database. Images containing a signal segment with more than $50 \%$ of its samples belonging to a certain rhythm were labelled as that specific rhythm.

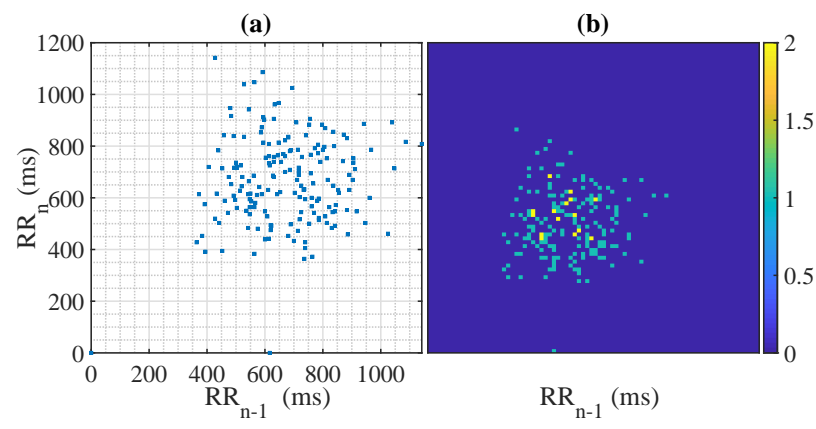

Figure 1.Example of image generation from a Poincaré plot. (a) Poincaré plot of a 2-min atrial fibrillation (AF) signal segment (b) the image generated from (a) using a bin size of 20x20 ms and an maximum extent of $1600 \mathrm{~ms}$, yielding a $80 \times 80$ pixels image.

\subsection{Atlas creation}

NSR, AF, and AB labelled images were extracted from all plots generated out of each ECG signal. All other images not labelled as one of these rhythms were discarded. Averaged images were constructed as reference images or atlases for each of the mentioned rhythms of study. Each atlas was defined as the average of the $80 \%$ of the images belonging to that specific rhythm, yielding three mean images representing NSR, $\mathrm{AF}$ and $\mathrm{AB}$ as shown in Figure 2.

\subsection{Mutual information analysis and clas- sification}

Mutual information, frequently used in medical image registration, measures the independence between two random variables. Likewise, it determines the amount of information one image contains about the other or how its uncertainty decreases when the other image is given. Mutual information can be expressed through diverse relations: regarding Shannon entropy, joint entropy or the KullballLeibler distance. Each definition contains the same information and can be rewritten into the others. Based on Shannon entropy, the mutual information between two images $\mathrm{A}$ and $\mathrm{B}, I(A, B)$, can be expressed as

$$
I(A, B)=H(B)-H(B \mid A),
$$

where $H(B)$ corresponds to the Shannon entropy of $B$ based on the probability distribution of its pixels' values. $H(B \mid A)$ instead represents the conditional entropy computed on the conditional probabilities $p(b \mid a)$ of pixels from image $A$ and $B$ [12].

Mutual information was computed between the remaining $20 \%$ of images of each rhythm category and each of the atlases. Based on the results obtained from the mutual information analysis, images were classified as one of the three rhythms (the chosen rhythm was the one with the highest mutual information value). To obtain robust results ten fold cross validation was performed, in which images used for constructing the reference and those used for the test, were randomly chosen. As a result, we obtained ten different average and test sets we performed the study with.

\section{Results}

\subsection{Images generated}

The total amount of images generated for the three rhythms is displayed in Table 1 . The third column of the table indicates the total number of different patients from which the images come from. Figure 2 displays an example of one of the ten average images generated with the $80 \%$ of the data for each of the three rhythms. 

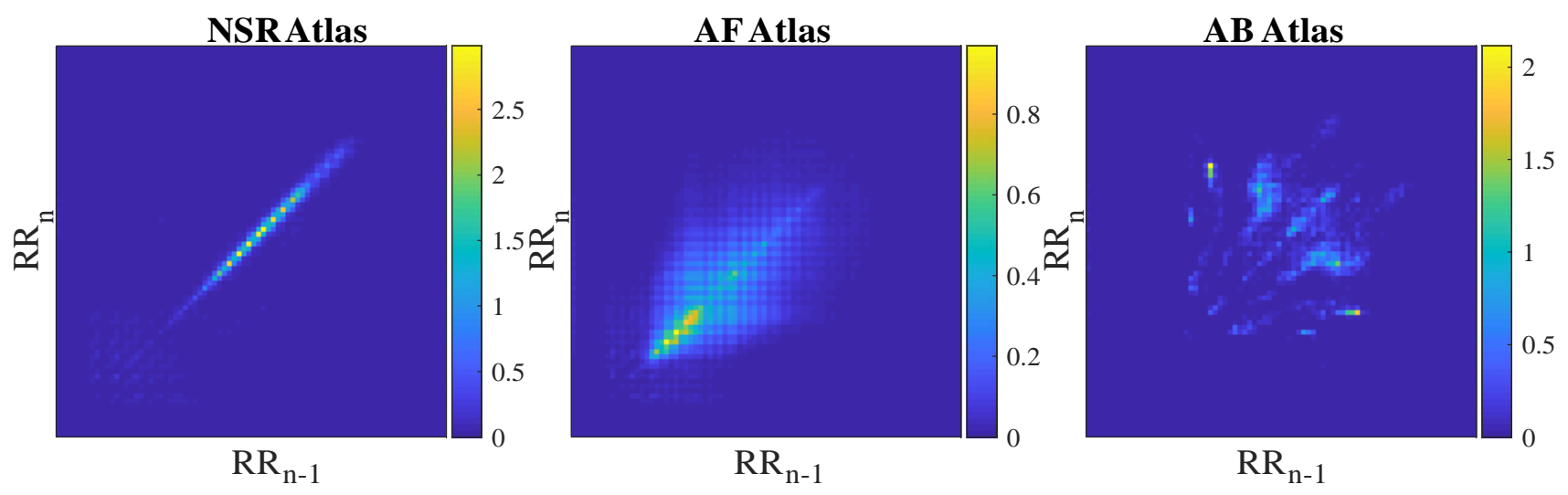

Figure 2.One of the 10 different sets of atlas images computed during the study representing normal sinus rhythm (NSR), atrial fibrillation $(\mathrm{AF})$ and atrial bigeminy $(\mathrm{AB})$.

Table 1. Total number of images used per rhythm and number of patients data were extracted from.

\begin{tabular}{lcr}
\hline \hline Rhythms & Total Images & Patients \\
\hline Normal sinus rhythm (NSR) & 27955 & 59 \\
Atrial Fibrillation (AF) & 33637 & 85 \\
Atrial Bigeminy (AB) & 76 & 6 \\
\hline \hline
\end{tabular}

\subsection{Classification}

The average results of the classification of the $20 \%$ of the images of each rhythm are displayed on Figure 3. In each subplot, each bar represents the percentage of images classified as NSR, AF, or AB, using mutual information between each of them and the references. It can be noted that from the 5591 NSR images used for testing, $80 \%$ were correctly classified as NSR while $18 \%$ were classified as $\mathrm{AF}$ and $2 \%$ as $\mathrm{AB}$.

$\mathrm{AF}$ images were classified correctly as $\mathrm{AF}$ in the $94 \%$ of the cases, as NSR in $5 \%$ and as $\mathrm{AB}$ in $1 \%$. Finally, $\mathrm{AB}$ was successfully categorized as AB in $72 \%$, as NSR in $2 \%$ and as $\mathrm{AF} 26 \%$ of the times.

\section{Discussion}

This study's main finding is that Poincaré plot-based images seems a promising way to classify different rhythms. It is worth noting that AF classification showed superior results compared to NSR and AB. In addition, only $0.53 \%$ of $\mathrm{AF}$ images were misclassified as $\mathrm{AB}$, meaning $\mathrm{AF}$ was successfully distinguished from $\mathrm{AB}$. On the other hand, about $20 \%$ of NSR segments were classified as AF. A reason for this misclassification could be the fact that signal segments used for generating Poincaré plots may contain multiple rhythms. Thus, the presence of short
AF episodes on signal segments labelled as NSR could have induced the misclassification.

Other Poincaré plot-based sensors using descriptive parameters, only focused towards AF detection, in [4] 91.4\% and $92.9 \%$ Se and Sp respectively values were obtained. In [9] a novel representation of Poincaré plot was presented obtaining $94.4 \%$ Se and $92.6 \%$ Sp percentages with a window of only 32 heart beats. AF detection in [7] reached a Sn of $97.5 \%$ and a Sp of $99 \%$ using a 2 min window. Atrial tachycardia (AT) was also targeted in this study but obtaining very low sensitivity; $92 \%$ of $\mathrm{Sp}$ and $57.7 \%$ of Sn. These results suggest parameter and threshold-based approaches are effective in monitoring a single arrhythmia but narrow down the ability to capture others, possibly due to the necessity of an explicit description of each rhythm and its particular behaviour. The presented reference-image approach gathers all characteristic rhythm-specific parameters implicitly and seems to enable the identification of different tachyarrhythmias by studying the $R R$ patterns as a whole. This also implies ectopic beats are integrated in the images without the necessity of explicitly applying measures or parameters for preventing their misclassification.

However, further research should be carried out using a wider database. The fact that all images were used either for creating the reference or for testing, independently of the patient, may have biased the results due to the underlying similarities between signal segments belonging to a same individual. In addition, the reduced amount of $A B$ images compared to those used for NSR and AF led to high standard deviations in the classification. A higher number of $\mathrm{AB}$ episodes and patients should be considered in order to acquire more accurate and significant results. 

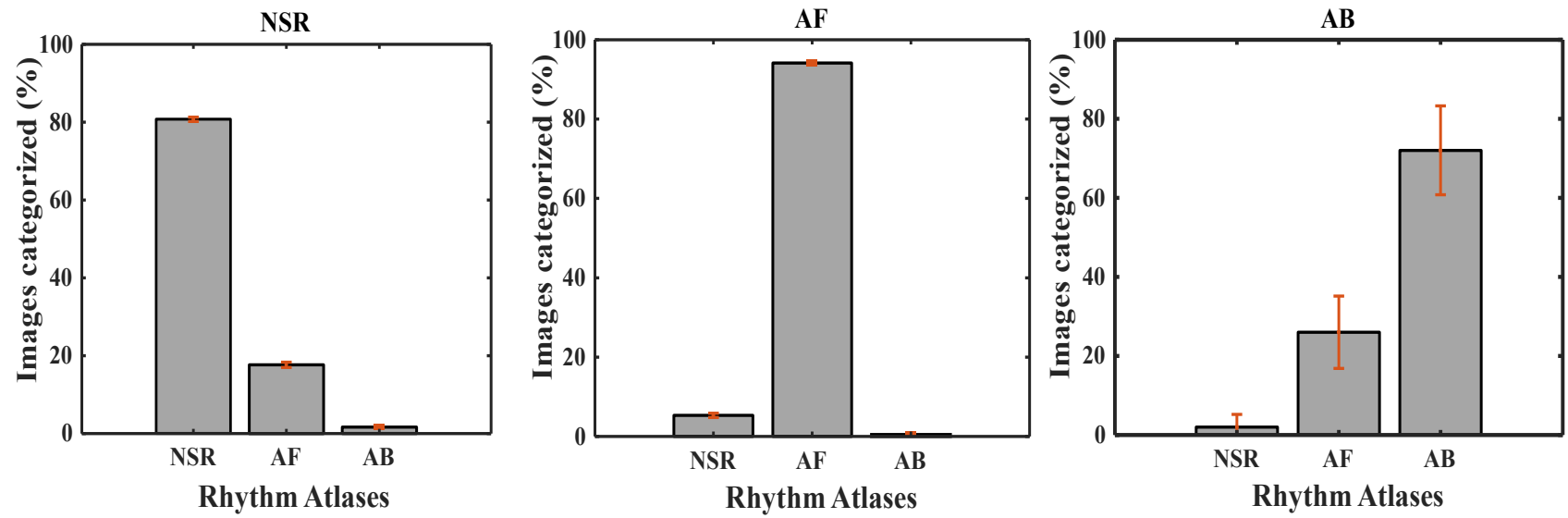

Figure 3.Classification results of the analysis after the 10 iterations. Each barplot represents in percentage, the distribution of the classification of all the images with a determined rhythm; normal sinus rhythm, (NSR), atrial fibrillation (AF) or atrial bigeminy $(\mathrm{AB})$, into one of the three rhythms.

\section{Conclusion}

The present paper suggest a different approach for rhythm classification using Poincaré plot-based images. The results of the proposed approach present a promising path for improvement and further study by the generation of a wider database including different rhythms, the tuning of the applied parameters (bin size, time window and time extent considered), and by applying different machine learning or even deep learning techniques able to extract implicit key features for an improved classification.

\section{Acknowledgments}

This project is framed inside MY-ATRIA consortium. MY-ATRIA project has received funding from the European Union's Horizon 2020 research and innovation programme under the Marie Skłodowska-Curie grant agreement No.766082

\section{References}

[1] Glotzer TV, Daoud EG, Wyse DG, Singer DE, Ezekowitz MD, Hilker C, Miller C, Qi D, Ziegler PD. The relationship between daily atrial tachyarrhythmia burden from implantable device diagnostics and stroke risk: the trends study. Circulation Arrhythmia and Electrophysiology 2009;2(5):474480.

[2] Bruser C, Diesel J, Zink MD, Winter S, Schauerte P, Leonhardt S. Automatic detection of atrial fibrillation in cardiac vibration signals. IEEE journal of biomedical and health informatics 2012;17(1):162-171.

[3] Lake DE, Moorman JR. Accurate estimation of entropy in very short physiological time series: the problem of atrial fibrillation detection in implanted ventricular devices. American Journal of Physiology Heart and Circulatory Physiology 2010;300(1):H319-H325.
[4] Park J, Lee S, Jeon M. Atrial fibrillation detection by heart rate variability in poincare plot. Biomedical engineering online 2009;8(1):38.

[5] Karmakar CK, Khandoker AH, Gubbi J, Palaniswami M. Complex correlation measure: a novel descriptor for poincaré plot. Biomedical engineering online 2009;8(1):17.

[6] Zhang L, Guo T, Xi B, Fan Y, Wang K, Bi J, Wang Y. Automatic recognition of cardiac arrhythmias based on the geometric patterns of poincaré plots. Physiological measurement 2015;36(2):283.

[7] Sarkar S, Ritscher D, Mehra R. A detector for a chronic implantable atrial tachyarrhythmia monitor. IEEE Transactions on Biomedical Engineering 2008;55(3):1219-1224.

[8] Monahan K, Song Y, Loparo K, Mehra R, Harrell FE, Redline S. Automated detection of atrial fibrillation from the electrocardiogram channel of polysomnograms. Sleep and Breathing 2016;20(2):515-522.

[9] Lian J, Wang L, Muessig D. A simple method to detect atrial fibrillation using rr intervals. The American journal of cardiology 2011;107(10):1494-1497.

[10] Goldberger AL, Amaral LA, Glass L, Hausdorff JM, Ivanov PC, Mark RG, Mietus JE, Moody GB, Peng CK, Stanley HE. Physiobank, physiotoolkit, and physionet: components of a new research resource for complex physiologic signals. Circulation 2000;101(23):e215-e220.

[11] Pan J, Tompkins WJ. A real-time qrs detection algorithm. IEEE Trans Biomed Eng 1985;32(3):230-236.

[12] Pluim JP, Maintz JA, Viergever MA. Mutual-informationbased registration of medical images: a survey. IEEE transactions on medical imaging 2003;22(8):986-1004.

Address for correspondence:

Guadalupe García Isla

Via Camillo Golgi 39, 20133 Milano, Italy

guadalupe.garcia@polimi.it 